\title{
Cauda Equina Paraganglioma: A Report of a Rare Case and Review of Literature
}

\author{
Mukesh Shukla $^{1}$ Jayesh Sardhara ${ }^{1}$ Kuntal Kanti Das ${ }^{1}$ Chaitanya Godbole $^{1}$ Sushila Jaiswal ${ }^{2}$ \\ Awadhesh Jaiswal $^{1}$ Sanjay Behari ${ }^{1}$ \\ ${ }^{1}$ Department of Neurosurgery, Sanjay Gandhi Postgraduate Institute \\ of Medical Sciences, Lucknow, Uttar Pradesh, India \\ 2 Department of Pathology, Sanjay Gandhi Postgraduate Institute of \\ Medical Sciences, Lucknow, Uttar Pradesh, India \\ Address for correspondence Awadhesh Jaiswal, MCh, Department of \\ Neurosurgery, 1st Floor C Block, Main Hospital Building, Sanjay \\ Gandhi Postgraduate Institute of Medical Sciences, Rae Bareilly Road, \\ Lucknow, Uttar Pradesh 226014, India \\ (e-mail: jaiswal_dr_2002@yahoo.co.in). \\ Indian J Neurosurg 2016;5:189-192.
}

\begin{abstract}
Keywords

- paraganglioma

- lumbar

- surgery

- pathology

- Extra-adrenal

Extra-adrenal paragangliomas (EAPs) are benign tumors of neuroendocrine origin. These tumors mostly arise from the carotid body and the jugular bulb area. EAP affecting the spine and filum terminale, known as cauda equine paraganglioma is rarely encountered in clinical practice. In this report, we present a case of a 28-year-old lady with paraganglioma at $\mathrm{L} 2 / 3$ level who presented with low back pain with radiation of pain to both legs for 4 year duration. The patient underwent $L 2 / 3$ laminoplasty with total excision of tumor. Following surgery, backache and radicular pain subsided completely. We discuss the case and present a review of other cases reported in the literature.
\end{abstract}

\section{Introduction}

Paragangliomas are benign tumors of neuroendocrine origin affecting the adrenal glands most frequently. Extra-adrenal paragangliomas (EAPs) are rare. Most EAPs are derived from carotid body or the jugular bulb. ${ }^{1}$ Paragangliomas affecting the spine are extremely rare. ${ }^{2}$ Spinal paragangliomas tend to affect the lumbar spine more often than other parts of the spine. Cauda equine paragangliomas (CEPs) can clinicoradiologically simulate nerve sheath tumors. Herein, we present the case of a 28-year-old lady with lumbago and lower limb radiculopathy resulting from paraganglioma at L2/3 level. We discuss the findings of the case and present a review of literature based on previously reported cases to gain some further insight into this uncommon entity.

\section{Case Description}

A 28-year-old lady presented with low back pain with radiation of pain to both lower limbs for 4 years. The pain used to be worsened by spinal movement and coughing. Sphincter function was normal. Local evaluation revealed tenderness in lumbar region, straight leg raising test of both legs produced posterior thigh pain at 30 degrees. Tendon reflexes, perianal sensitivity, and anal tone were normal. Magnetic resonance imaging (MRI) of the lumbosacral spinal segment demonstrated an oval-shaped intradural extramedullary lesion measuring $4 \times 1.5 \times 1.2 \mathrm{~cm}$ at L2-L3 level. The lesion was isointense on $\mathrm{T} 1$-weighted and heterogeneously hyperintense on T2-weighted images. There was a marked contrast enhancement after gadolinium administration (-Fig. 1a-d). A standard L2-L3 laminoplasty was performed to gain access to the intradural lesion. The lesion was then removed "en bloc" via a standard duratomy (-Fig. 1e). The mass had the appearance of an irregular-shaped nodule of the dimension previously indicated by MRI. The tumor was soft, round, well-encapsulated and vascularized, bluish red in color, and contiguous with the spinal root but not adhered to any other structures. During "en bloc" removal, the nerve root was resected cranially to the lesion, bluntly dissecting it from the caudal roots which appeared displaced but showed no evidence of infiltration. The radicular lower extremity pain resolved received

December 29, 2015

accepted

January 18, 2016

published online

June 24, 2016
DOI http://dx.doi.org/

10.1055/s-0036-1581988. ISSN 2277-954X.
(C) 2016 Neurological Surgeons' Society of India
License terms

(요 (1) $\Theta \circledast$ 

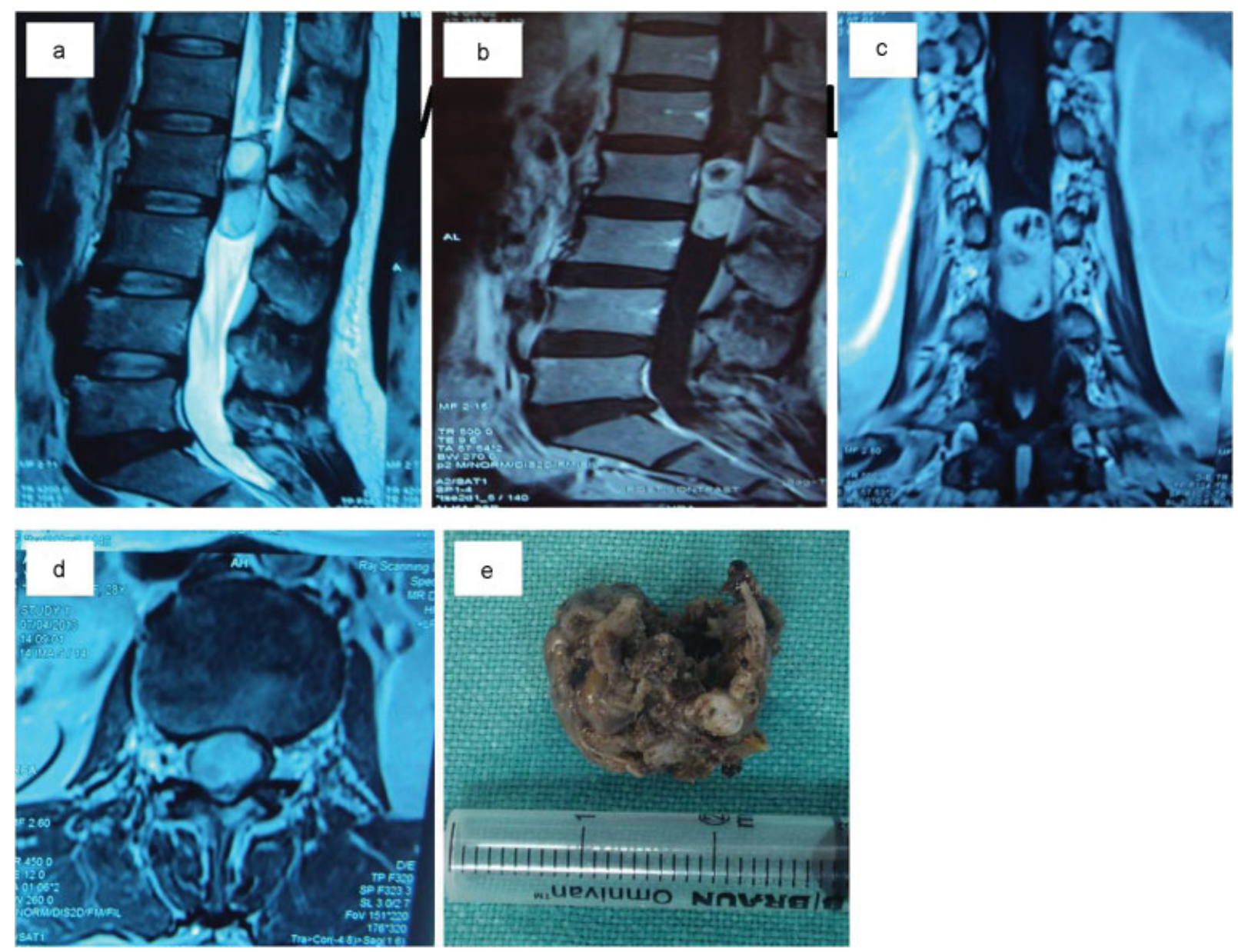

Fig. 1 (a) A predominantly T2-hyperintense vertically elongated intradural extramedullary mass from upper border of L2 to midportion of $L 3$ body shifting the nerve roots posteriorly. (b-d) Intense heterogeneous enhancement of the lesion with few necrotic areas. (e) The resected specimen.

completely after surgery. Histological evaluation of the tumor showed uniform cells arranged either in large lobules, or smaller nests. The aggregated chief cells were surrounded by capillaries, present throughout the lesion. There was a flattened layer of sustentacular cells, positive for S100 which encompassed both the lobules and the nests. Immunohistochemical examination gave a positive reaction for cytokeratin, synaptophysin, and chromogranin in the chief cells (-Fig. 2a-d).

The patient had an uneventful recovery after surgery and his preoperative symptoms of pain and radiculopathy completely resolved. As the lesion was completely excised, no further treatment was advised. She was doing well and pain free at 8 months follow-up.

\section{Discussion}

Paraganglioma is a neuroendocrine tumor derived from the chromaffin cells of neural crest origin. ${ }^{3}$ The first authors to describe this pathological entity were Miller and Torack in 1970 , denominating it a secretory ependymoma, ${ }^{4}$ whereas Lerman et al were the first to coin the term paraganglioma of the cauda equina in 1972. The great majority of paragangliomas (85-90\%) arise in the adrenal gland. EAPs are usually located in the head and neck region, with $90 \%$ arising in the carotid body or glomus jugulare. EAPs have been reported in various diverse locations, such as prostate, larynx, gall bladder, intrathoracic region, and spine. ${ }^{5}$ Extraspinal paragangliomas are usually multiple and have a higher association with syndromes such as Von Hippel Lindau disease and multiple endocrine neoplasia II syndrome. In the central nervous system, the most common extraspinal localizations of paraganglioma are the petrous ridge, pineal gland, and sella turcica. ${ }^{6}$ The spinal localization is uncommon, with an estimated incidence in the general population calculated at $0.07 / 100,000$ inhabitants. Spinal paragangliomas are mostly encountered in the cauda equina and the filum terminale region. These may, occasionally, be associated with syringomyelia ${ }^{3}$ a or intramedullary cyst. ${ }^{1}$ Yang et al noted only 3 patients of CEPs out of 105 intradural extramedullary operated at their center over 11 years. ${ }^{7}$ Unlike head and neck paragangliomas, spinal paragangliomas tend to be typically sympathetic and typically nonsecretory.

Apart from the sympathetic neurons in the lateral horn, other possible origins of spinal paragangliomas include heterotopic neurons lying along the branches proximal to the sympathetic trunk, paraganglia situated at cauda equine, and very rarely from differentiation of local ependymal., ${ }^{8,9}$

Although these tumors are known to be benign, local recurrence, and malignant degenerations are known to occur (12 and 6.5\%, respectively). This particularly applies for EAPs. ${ }^{5}$ 


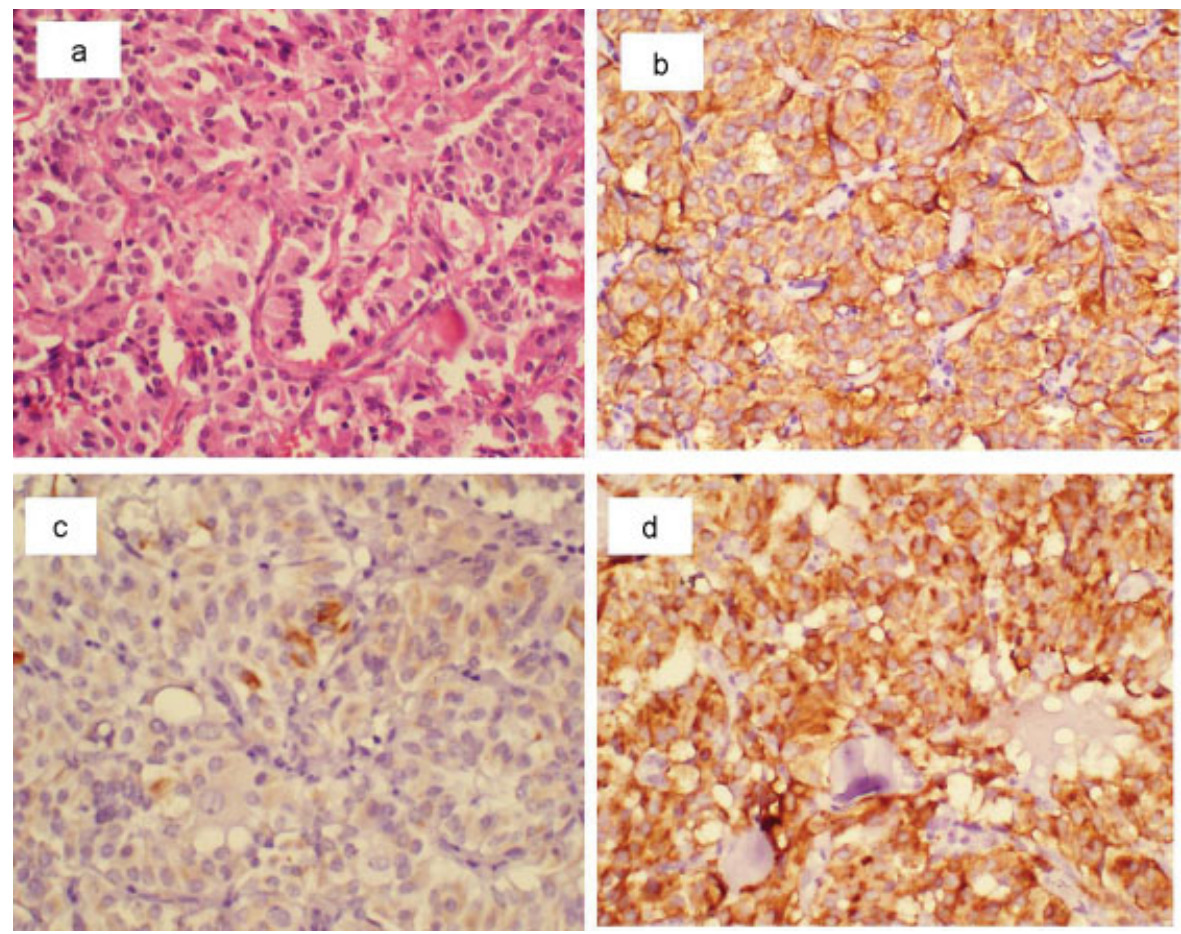

Fig. 2 H\&E stained (a) section shows tumor disposed in nests and lobules displaying uniform round to polygonal cells with central nuclei, stippled chromatin, inconspicuous nucleoli, and moderate amount of granular eosinophilic cytoplasm $(\times 20)$. On immunohistochemistry tumor cells are positive for (b) synaptophysin $(\times 40)$, (c) cytokeratin, and (d) chromogranin. H\&E, hematoxylin and eosin.

Reports suggest that usual MR features of paragangliomas are hardly diagnostic of this condition and may mimic other intradural extramedullary lesions such as schwannoma and meningioma. ${ }^{10}$ In fact, imaging features in our patient gave an impression of nerve sheath tumor. The hypervascular nature of paragangliomas, however, is reported to be a distinctive feature. This hypervascularity results in "salt-and-pepper" appearance on T2-weighted images that may sometimes be helpful in diagnosis especially when the mass is large enough. ${ }^{11}$ However, we did not observe this sign in our patient. Conventional angiography demonstrates an intense early blush that persists into the late arterial and early venous phases. However, conventional DSA is hardly recommended for intradural extramedullary lesions of this area. In the interpretation of MRI, following two observations may be helpful in diagnosis of paraganglioma: (1) a defect in the serpiginous structure between the conus and the lesion, suggesting dilatation of the serpentine vessels, which is uncommon in schwannomas and ependymomas. (2) A T2-hypointense tumor rim suggesting hemosiderin deposits, so very characteristic of vascular tumors. ${ }^{10,11}$ As suggested by Yang et al, radiologically, these tumors masquerade as other intradural extramedullary masses such as schwannoma and meningioma and only histopathology is able to provide a decisive diagnosis.?

On histological examination, round-to-oval chief cells and the sustentacular or supporting cells make the most of the tumor. Histopathological diagnosis is based on the characteristics typical of paraganglioma, namely, an organoid or "Zellballen." The paucity or absence of sustentacular cells has been demonstrated to be an indicator of aggressive or malignant nature. ${ }^{12}$ Immunohistochemistry shows positivity for S100, NSE, CHR, and (S100, Neuron Specific Enolase, Chromogranin, and Synaptophysin, respectively). ${ }^{12,13}$ Electron microscopy also helps in making a histological diagnosis.

Surgical treatment aims at complete excision of the lesion that is usually well encapsulated, as in the case we observed. Complete excision is usually curative. Subtotal excision expectedly, is associated with recurrence.

The prognosis of these lesions is usually good, as long as they are completely removed. In fact, subtotal excision is frequently followed by local recurrence and even distant metastatic spread has also been described. ${ }^{8}$ On the other hand, vertebral metastases with spinal cord compression from carotid body and retroperitoneal paragangliomas were reported. Consequently, prognosis is better in lesions that are completely excised. Yang et al showed lack of recurrence following complete excision in all three patients they operated. ${ }^{7}$ When complete removal is not feasible, radiotherapy may be considered, although resistance to this form of treatment has been documented. ${ }^{13}$ Chemotherapy does not appear to play a role in the management of these lesions. The median free interval between surgical removal and local recurrence is estimated to be approximately 6 years. ${ }^{14}$ However, a longterm follow-up is mandatory. ${ }^{14}$ 


\section{Conclusions}

CEP is an uncommon condition, but mimics other intradural extramedullary lesions clinicoradiologically. Surgical finding of a reddish firm mass may often provide a clue. The goal of surgical treatment is total resection of the lesion. Patients in whom complete removal is not feasible, assiduous long-term follow-up is required. Although these tumors are nonsecretory and hardly require any additional workup, we would recommend considering paraganglioma as a differential diagnosis of intradural extramedullary lesions of conus-cauda region.

\section{Consent Statement}

A written informed consent was obtained from the patient for publication of this case report and any accompanying images.

\section{Competing Interests}

The authors have not been influenced by any financial or personal relationship with people or organizations in preparation of this study.

\section{References}

1 Faro SH, Turtz AR, Koenigsberg RA, Mohamed FB, Chen C-Y, Stein H. Paraganglioma of the cauda equina with associated intramedullary cyst: MR findings. AJNR Am J Neuroradiol 1997;18(8):1588-1590

2 Singh RV, Yeh JS, Broome JC. Paraganglioma of the cauda equina: a case report and review of the literature. Clin Neurol Neurosurg 1993;95(2):109-113
3 Steel TR, Botterill P, Sheehy JP. Paraganglioma of the cauda equina with associated syringomyelia: case report. Surg Neurol 1994; 42(6):489-493

4 Lerman RI, Kaplan ES, Daman L. Ganglioneuroma-paraganglioma of the intradural filum terminale. Case report. J Neurosurg 1972; 36(5):652-658

5 Fitzgerald LF, Cech DA, Goodman JC. Paraganglioma of the thoracic spinal cord. Clin Neurol Neurosurg 1996;98(2): 183-185

6 Kruse F Jr. Petrous ridge chemodectoma (nonchromaffin paraganglioma) simulating meningioma. Case report. J Neurosurg 1960;17:1108-1111

7 Yang SY, Jin YJ, Park SH, Jahng TA, Kim HJ, Chung CK. Paragangliomas in the cauda equina region: clinicopathoradiologic findings in four cases. J Neurooncol 2005;72(1):49-55

8 Sonneland PR, Scheithauer BW, LeChago J, Crawford BG, Onofrio BM. Paraganglioma of the cauda equina region. Clinicopathologic study of 31 cases with special reference to immunocytology and ultrastructure. Cancer 1986;58(8):1720-1735

9 Miller CA, Torack RM. Secretory ependymoma of the filum terminale. Acta Neuropathol 1970;15(3):240-250

10 Levy RA. Paraganglioma of the filum terminale: MR findings. Am J Roentgenol 1993;160(4):851-852

11 van Gils AP, van den Berg R, Falke TH, et al. MR diagnosis of paraganglioma of the head and neck: value of contrast enhancement. AJR Am J Roentgenol 1994;162(1):147-153

12 Kliewer KE, Cochran AJ. A review of the histology, ultrastructure, immunohistology, and molecular biology of extra-adrenal paragangliomas. Arch Pathol Lab Med 1989; 113(11):1209-1218

13 Yang SY, Jin YJ, Park SH, Jahng TA, Kim HJ, Chung CK. Paragangliomas in the cauda equina region: clinicopathoradiologic findings in four cases. J Neurooncol 2005;72(1):49-55

14 Say CC, Hori J, Spratt J Jr. Chemodectoma with distant metastasis: case report and review of the literature. Am Surg 1973;39(6): 333-341 